\title{
LOCAL CONTRACTIONS OF COMPACT METRIC SETS WHICH ARE NOT LOCAL ISOMETRIES
}

\author{
R. F. WILLIAMS
}

Following Albert Edrei [1], if $X$ is a compact metric space with metric $\rho, f$ is a mapping of $X$ onto $X$, and $x \in X$, then $x$ is said to be a point of contraction under $f$ relative to $X$ provided that there is a positive number $\mu(x)$ such that if $y \in X$ and $\rho(x, y)<\mu(x)$, then $\rho[f(x), f(y)] \leqq \rho(x, y)$. Further, if each point of $X$ is a point of contraction under $f$ relative to $X, f$ will be said to be a local contraction of $X$. Edrei posed the following question: if $X$ is a compact metric space and $f$ is a contraction of $X$ onto $X$, is $f$ a local isometry? The purpose of this paper is to answer this question in the negative.

1. Basic example. Throughout this section polar coordinates, $(r, \theta)^{\prime}$ are used, $W$ denotes the origin, $\phi$ denotes the rotation defined by $\phi(r, \theta)=(r, \theta+1)$, and $\rho$ denotes the Euclidean distance function. Let $R=10 / 9$, and for each non-negative integer $i$, let $R_{i}=\sum_{j=0}^{i} 1 / 10^{i}$, and $Q_{i}=\left(R_{i}, 0\right)$. Let $m_{0}=n_{0}=0$ and $P_{0}=Q_{0}$. There exists a positive integer $n_{1}$ such that $\rho\left[\phi^{n_{1}}\left(P_{0}\right), Q_{0}\right]<.1$. Let $m_{1}=n_{1}, P_{m_{1}}^{\prime}=\phi^{m_{1}}\left(P_{0}\right)$, and for each integer $i, n_{0}<i<n_{1}$, let $P_{i}=\phi^{i}\left(P_{0}\right)$. Let $A_{1}^{\prime}$ be the polar angle of $P_{m_{1}}^{\prime}$ and let $A_{1}$ denote an angle such that $\cos A_{1}=\left(R_{0} / R_{1}\right) \cos A_{1}^{\prime}$. Let $P_{m_{1}}=\left(R_{1}, A_{1}\right)$.

There exists a positive integer $n_{2}$ such that $\rho\left[\phi^{n_{2}}\left(P_{m_{1}}\right), Q_{1}\right]<.01$. Let $m_{2}=m_{1}+n_{2}$, let $P_{m_{2}}^{\prime}=\phi^{n_{2}}\left(P_{m_{1}}\right)$, and for each integer $i, n_{0}<i<n_{2}$, let $P_{m_{1}+i}=\phi^{i}\left(P_{m_{1}}\right)$. Let $A_{2}^{\prime}$ be the polar angle of $P_{m_{2}}^{\prime}$, and let $A_{2}$ denote an angle such that $\cos A_{2}=\left(R_{1} / R_{2}\right) \cos A_{2}^{\prime}$. Let $P_{m_{2}}=\left(R_{2}, A_{2}\right)$.

Continuing this process indefinitely we obtain: (1) two sequences $\left\{n_{i}\right\}_{i=1}^{\infty},\left\{m_{i}\right\}_{i=1}^{\infty}$ of positive integers; (2) two sequences $\left\{P_{m}\right\}_{m=0}^{\infty}$, $\left\{P_{m_{i}}^{\prime}\right\}_{i=1}^{\infty}$ of points; and (3) two sequences $\left\{A_{i}^{\prime}\right\}_{i+1}^{\infty},\left\{A_{i}\right\}_{i=1}^{\infty}$ of angles. The elements thus obtained have the following properties:

(1) $m_{i+1}=m_{i}+n_{i+1}$;

(2) $\rho\left(P_{m_{i+1}}^{\prime}, Q_{i}\right)<1 / 10^{i}$;

(3) $P_{m_{i}+j}=\phi^{i}\left(P_{m_{i}}\right)$, for $n_{0}<j<n_{i+1}$;

(4) $P_{m_{i+1}}^{\prime}=\phi^{n_{i+1}}\left(P_{m_{i}}\right)=\left(R_{i}, A_{i+1}^{\prime}\right)$;

(5) $P_{m_{i+1}}=\left(R_{i+1}, A_{i+1}\right)$;

(6) $\cos A_{i+1}=\left(R_{i} / R_{i+1}\right) \cos A_{i+1}^{\prime}$.

Let $C$ denote the circle, $r=10 / 9, Q=(10 / 9,0), K=\bigcup_{n=0}^{\infty} P_{n}$, $M=C \cup K$, and $L=\bigcup_{i=1}^{\infty} P_{m_{i}}$. The sequence $\left\{P_{m_{i}}\right\}$ converges to $Q$.

Presented to the Society, June 20, 1953; received by the editors May 2, 1953 and, in revised form, December 14, 1953. 
For since $\left\{Q_{i}\right\}$ converges to $Q$, so does $\left\{P_{m_{i}}^{\prime}\right\}$. Therefore $\left\{\cos A_{i}^{\prime}\right\} \rightarrow 1$ and so $\left\{\cos A_{i}\right\}=\left\{\left(R_{i-1} / R_{i}\right) \cos A_{i}^{\prime}\right\} \rightarrow 1$.

Let $f$ denote the transformation defined by

$$
f(P)= \begin{cases}\phi^{-1}(P), & \text { if } P \in C ; \\ P_{i}, & \text { if for some non-negative } i, P=P_{i+1} ; \\ P_{0}, & \text { if } P=P_{0} .\end{cases}
$$

Thus if $P \in M-L, f(P)=\phi^{-1}(P)$. Therefore $f$ is a local isometry at all points of $M-Q$. To show that $f$ is a local contraction but not a local isometry, it will suffice to show that for each non-negative integer $i, \rho\left[f\left(P_{n_{i+1}}\right), f(Q)\right]<\rho\left(P_{n_{i+1}}, Q\right)$. But $\rho\left[f\left(P_{n_{i+1}}\right), f(Q)\right]$ $=\rho\left[\phi^{-1}\left(P_{n_{i+1}}^{\prime}\right), \phi^{-1}(Q)\right]=\rho\left(P_{n_{i+1}}^{\prime}, Q\right)<\rho\left(P_{n_{i+1}}, Q\right)$, the inequality following from the polar distance formula and relations (4), (5), and (6) above.

2. Other examples. In the example above, $f\left(P_{1}\right)=f\left(P_{0}\right)=P_{0}$ and so $f$ is not a homeomorphism. For each integer $i \leqq 2$ let $B_{i}=(1 / i, 0)$. Let $M^{\prime}=M \cup W \cup\left(\cup_{n=2}^{\infty} B_{n}\right)$, let $f^{\prime}=f$ on $M-P_{0}$, let $f^{\prime}\left(P_{0}\right)=B_{2}$, let $f^{\prime}\left(B_{i}\right)=B_{i+1}$, and let $f^{\prime}(W)=W$. Then $M^{\prime}$ is a compact set in the plane and $f^{\prime}$ is a homeomorphism of $M^{\prime}$ onto $M^{\prime}$ which is a local contraction but not a local isometry.

Using cylindrical coordinates, $(r, \theta, z)$, consider the plane of the basic example to be the graph of $z=1$, and consider $\phi$ to be the rotation defined by $\phi[(r, \theta, z)](r, \theta+1, z)$. Let $M^{\prime}$ be the cone over $M$ with vertex $(0,0,0)$, i.e., $M^{\prime}=\{(r z, \theta, z) \mid z \in[0,1]$ and $(r, \theta, 1) \in M\}$. Let $f^{\prime}$ denote the linear extension of $f$, i.e., $f^{\prime}[(z r, \theta, z)]=\left(z r^{\prime}, \theta^{\prime}, z\right)$ where $f(r, \theta, 1)=\left(r^{\prime}, \theta^{\prime}, 1\right)$. To show that $f^{\prime}$ is a local contraction of $M^{\prime}$ onto $M^{\prime}$, let $L_{n}=\left\{(z r, \theta, z) \mid z \in[0,1]\right.$ and $\left.(r, \theta, 1)=P_{n}\right\}$. Then for each integer $i \geqq 0, L_{i}$ is a line interval intersecting the closure of $M^{\prime}$ $-L_{i}$ only in the point $(0,0,0), f^{\prime}=\phi$ on $M^{\prime}-\bigcup_{i=0}^{\infty} L_{m_{i}}$, and each point of $L_{0}$ is fixed. As, for each $i \geqq 0, f^{\prime}$ is linear on $L_{m_{i+1}}$ and as $L_{m_{i+1}}$ and $f^{\prime}\left(L_{m_{i+1}}\right)=L_{m_{i+1}-1}$ have lengths $\left(1+R_{i+1}^{2}\right)^{1 / 2}$ and $\left(1+R_{i}^{2}\right)^{1 / 2}$ respectively, each point of $L_{m_{i+1}}$ is a point of contraction under $f^{\prime}$. As $\lim _{i \rightarrow \infty} L_{m_{i}}=L_{Q}=\{(z R, 0, z) \mid z \in[0,1]\}$, it will suffice to show that if $P \in L_{m_{i+1}}-(0,0,0)$ and $P^{\prime} \in L_{Q}$, then $\rho\left[f^{\prime}(P), f^{\prime}\left(P^{\prime}\right)\right]<\rho\left(P, P^{\prime}\right)$. Then let $P=\left(z R_{i+1}, A_{i+1}, z\right), z \neq 0$, and $P^{\prime}=(y R, 0, y) ; f^{\prime}(P)$ $=\left(z R_{i}, A_{i+1}^{\prime}, z\right)$ and $f^{\prime}\left(P^{\prime}\right)=(y R,-1, y)$. Therefore $\rho\left[f^{\prime}(P), f^{\prime}\left(P^{\prime}\right)\right]$ $=\rho\left[\left(z R_{i}, A_{i+1}^{\prime}-1, z\right),(y R,-1, y)\right]=\rho\left[\left(z R_{i}, A_{i+1}^{\prime}, z\right),(y R, 0, y)\right]$ $<\rho\left(P, P^{\prime}\right)$, the inequality following from the cylindrical distance formula and the relations (4), (5), (6) above and $z \neq 0$. Thus $f^{\prime}$ is a local contraction of a compact continuum onto itself which is not a local isometry. 
There exists a totally disconnected compact metric set $C$ and an isometry $f$ of $C$ onto $C$ such that for each point $x \in C$, the iterations $f(x), f^{2}(x), f^{3}(x), \cdots$, are dense in $C$. (E.g., see Vietoris [2], and let the metric $\rho$ be defined, using the triadic system, as follows:

$$
\begin{array}{llll}
\text { for } 0 \leqq x \leqq 0.1, & 0.2 \leqq y \leqq 1, & \rho(x, y)=1 / 2 ; \\
\text { for } 0 \leqq x \leqq 0.01, & 0.02 \leqq y \leqq 0.1, & \rho(x, y)=1 / 4 ; \\
\text { for } 0.2 \leqq x \leqq 0.21, & 0.22 \leqq y \leqq 1, & \rho(x, y)=1 / 4 ; \\
\text { for } 0 \leqq x \leqq 0.001, & 0.002 \leqq y \leqq 0.01, & \rho(x, y)=1 / 8 \\
\ldots .) &
\end{array}
$$

There exists a countable sequence of points $\left\{P_{i}\right\}$ in $C \times[0,1]$ which converges to a subset of $C_{0}$ (the "base set" in $\left.C \times[0,1]\right)$ in a manner completely analogous to the convergence of $\left\{P_{n}\right\}$ in the basic example. Let $M^{\prime}=C_{0} \cup\left(\cup_{i=0}^{\infty} P_{i}\right)$ and let $f^{\prime}$ be defined in a manner similar to $f$ in the basic example. Then $f^{\prime}$ is a local contraction of a totally disconnected compact metric set onto itself which is not a local isometry. $M^{\prime}$ and $f^{\prime}$ in these last two examples can be extended as above to yield homeomorphisms.

\section{BiBLIOGRAPHY}

1. Albert Edrei, On mappings which do not increase small distances, Proc. London Math. Soc. (3) vol. 2 (1952) p. 272.

2. L. Vietoris, Über den höheren Zusammenhang kompakter Räume und eine Klasse von zusammenhangstreuen Abbildung, Math. Ann. vol. 97 (1927) p. 459.

UNIVERSITY OF VIRGINIA 